IdeAs

Idées d'Amériques

$2 \mid 2012$

Universités d'Amériques et construction des savoirs

\title{
Les campi brésiliens
}

João Sette Whitaker Ferreira et Capucine Boidin

\section{(2) OpenEdition}

Journals

Édition électronique

URL : https://journals.openedition.org/ideas/346

DOI : 10.4000/ideas.346

ISSN : 1950-5701

\section{Éditeur}

Institut des Amériques

Référence électronique

João Sette Whitaker Ferreira et Capucine Boidin, «Les campi brésiliens », IdeAs [En ligne], 2 | 2012, mis en ligne le 22 juin 2012, consulté le 19 octobre 2022. URL : http://journals.openedition.org/ideas/346 : DOI : https://doi.org/10.4000/ideas.346

Ce document a été généré automatiquement le 19 octobre 2022

\section{(c) $($ i) $(9)$}

Creative Commons - Attribution - Pas d'Utilisation Commerciale - Pas de Modification 4.0 International - CC BY-NC-ND 4.0

https://creativecommons.org/licenses/by-nc-nd/4.0/ 


\title{
Les campi brésiliens
}

\author{
João Sette Whitaker Ferreira et Capucine Boidin
}

Au Brésil, les bâtiments universitaires étaient d'abord situés en centre ville, puis s'est diffusé le modèle des "campi", à l'extérieur de la ville ; un processus qui n'est pas étranger à l'histoire récente des universités en France. Pouvez nous dire ce que cela signifie à l'échelle du Brésil ?

João Sette Whiteker Ferreira : Les campi universitaires au Brésil sont surtout marqués par leur diversité spatiale. Au début du $\mathrm{xx}^{\mathrm{e}}$ siècle les premières universités proposaient des cours supérieurs de médecine ou de droit (faculté de médecine de Bahia en 1808, de droit à Olinda et à São Paulo en 1827), puis d'ingénieurs. Chacun dans leur contexte historique, ces formations supérieures furent instaurées soit pour désigner le Brésil comme nouveau centre de l'empire Portugais en $1808^{1}$, soit pour affirmer l'indépendance et la jeune république, ou encore, au début du $x^{\mathrm{e}}$ siècle, pour donner un support technique et scientifique aux activités agro-exportatrices (ingénierie pour les chemins de fer, par exemple). Dans tous les cas, ce furent des bâtiments isolés, imposants, souvent au centre ville, chargés d'un fort symbolisme.

D'une manière générale, il y a trois types de campi universitaires au Brésil, qui correspondent assez bien aux trois modalités d'universités elles-mêmes. Les universités publiques tout d'abord, elles sont soit fédérales et éparpillées dans tout le pays, soit étatiques. À l'exception des bâtiments des universités historiques commentés ci-dessus, ces universités publiques ont bénéficié de la possibilité $\mathrm{d}$ 'expropriation de grands terrains pour leur installation, et sont en général installées dans des campi «à l'américaine », très amples ${ }^{2}$. Les universités fédérales créées par le gouvernement Lula suivent le même schéma. Dans les grandes régions métropolitaines, cependant, les nouveaux campi fédéraux ne peuvent plus bénéficier de grands terrains expropriés, en raison de la forte valorisation foncière et du manque de disponibilité de terres. Dans ce cas, comme pour la nouvelle université fédérale de l'ABC (région métropolitaine de São Paulo), le campus est un seul et très grand immeuble, ou un groupe de deux ou trois bâtiments, sur des aires d'environ 50 mille mètres carrés. Je reparlerai plus loin de ce type de campus, avec l'exemple de l'université de São Paulo, l'USP. 
Le deuxième type d'université, qui est aussi assez ancien, est celui des institutions dites « communautaires » ou " confessionnelles ", qui sont liées à des ordres religieux ou à des fondations. Il y a des universités très importantes et traditionnelles dans ce groupe, comme la Pontificale université de São Paulo - PUC, ou celle de Rio (il y a des PUC dans beaucoup de villes brésiliennes), de l'église catholique, ou encore 1 'université Mackenzie, à São Paulo, liée à l'église presbytérienne d'origine nordaméricaine, ou encore les universités de la fondation Getulio Vargas, à São Paulo ou Rio. Étant privées (mais à buts non lucratifs), ces universités, même anciennes (souvent crées dans les années 1940 ou 1950), n'ont pas reçu de terrains publics et sont, en général, situées sur des campi urbains, insérés dans la ville. Ainsi, Mackenzie, à São Paulo, occupe un grand quadrilatère dans une région assez centrale, tout comme la PUC. Le campus de Mackenzie est constitué d'une bonne dizaine de bâtiments, qui sont distribués d'une façon très urbaine et intéressante, car créant des rues internes au campus dans un quartier privilégié de la ville. On y trouve des commerces (alimentation...) et des services (banque). Bien qu'offrant une bonne continuité spatiale par rapport aux rues voisines, les contraintes de « sécurité » ont fait que le campus soit peu à peu fermé par des grilles et des portails. Ces mesures sont, en fait, issues d'une vision assez erronée de ce que peut être la sécurité urbaine, qui justement ne comprend pas l'importance de l'ouverture sur la ville et la création de flux urbains assez intenses et vifs. L'installation de barrières semblables à celles du métro, n'autorisant le passage qu'aux porteurs de cartes électroniques, est prévue pour cette année, malheureusement.

Campus université presbytérienne Mackenzie, São Paulo, Brésil

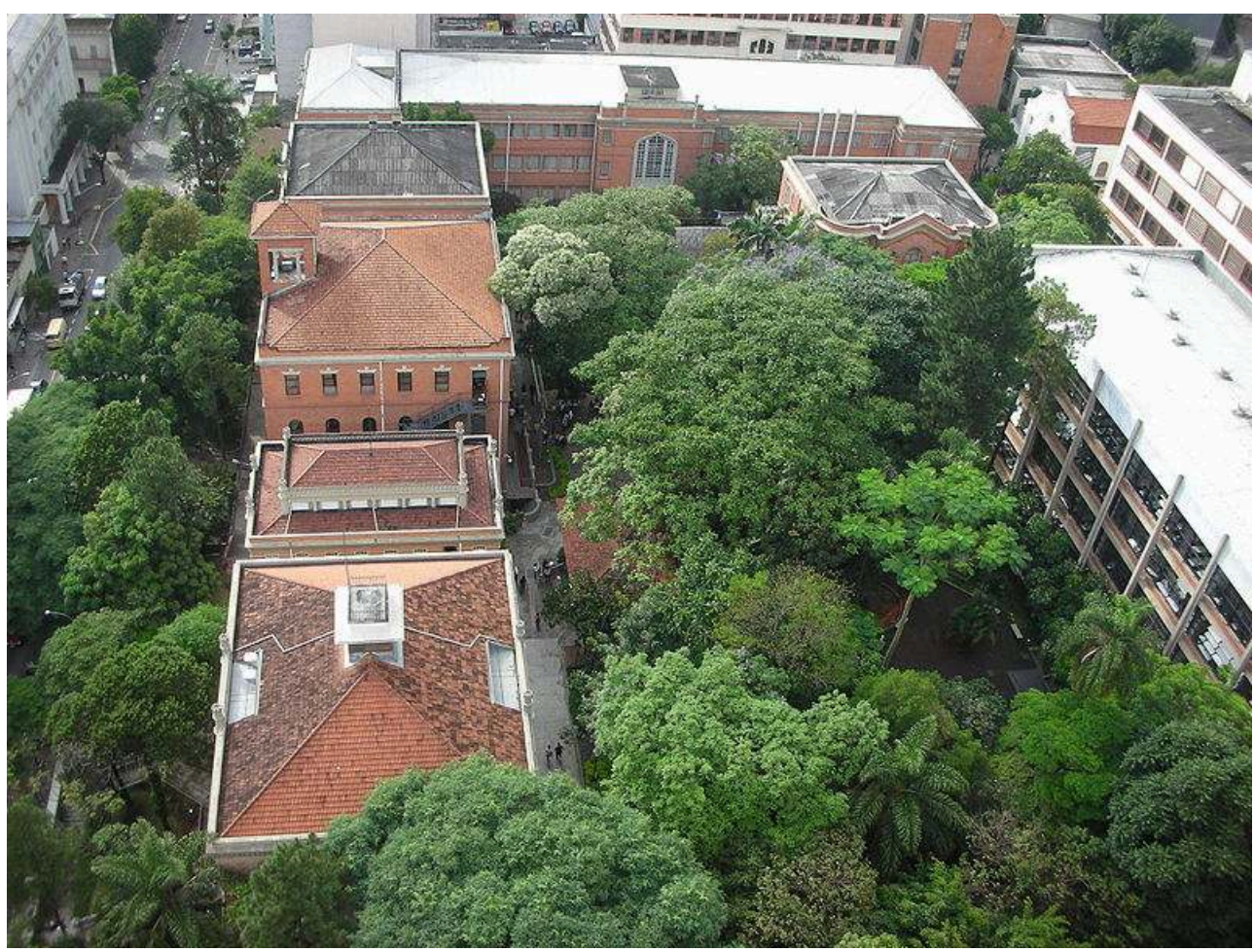

FABCOELHO - PUBLIC DOMAIN - CREATIVE COMMONS

Le campus principal de la PUC de São Paulo (il y en a d'autres plus petits) est aussi dans un quartier résidentiel assez central. Plus petit que celui du Mackenzie, il abrite 
en fait un immeuble ancien (ancien monastère), et un grand immeuble plus neuf de 6 étages, construit dans les années 1960. Il est aussi très urbain, et l'espace entre les deux immeubles crée une place très animée, avec les terrasses des étages de 1 'immeuble neuf qui sont tournées vers elle. Le campus de la PUC a une histoire intéressante, puisque lors de la dictature militaire, l'université était un bastion de résistance, embauchant grand nombre de professeurs expulsés des universités publiques. En 1977, il a été envahi par les troupes de la dictature, et le campus en est devenu un symbole de la lutte pour la démocratie.

Campus Pontificale université catholique de São Paulo de jour, Brésil

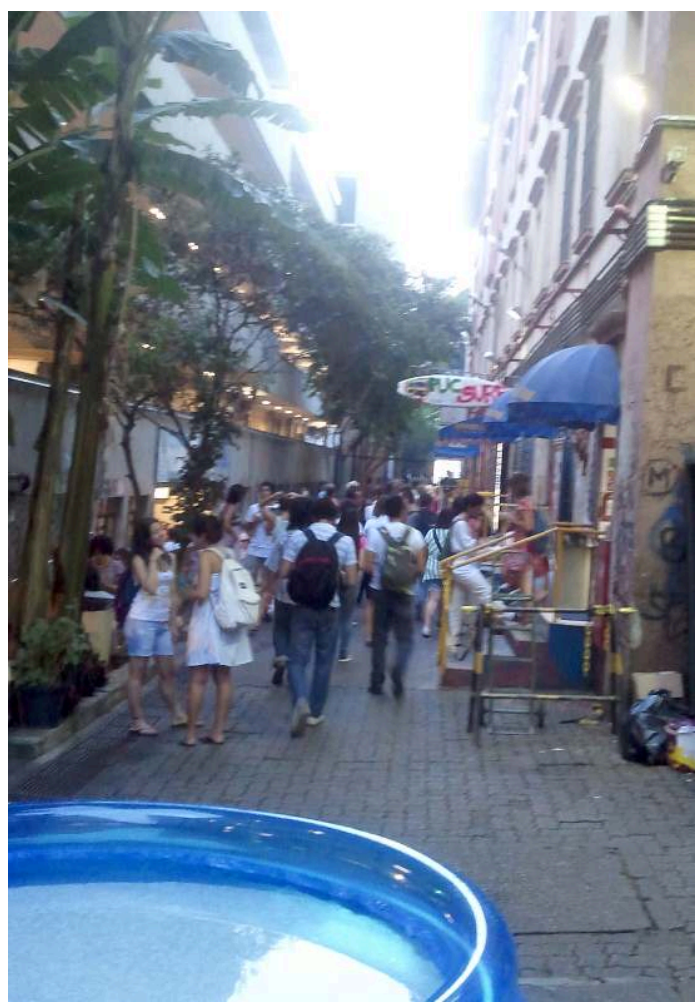

João Sette W. Ferreira 


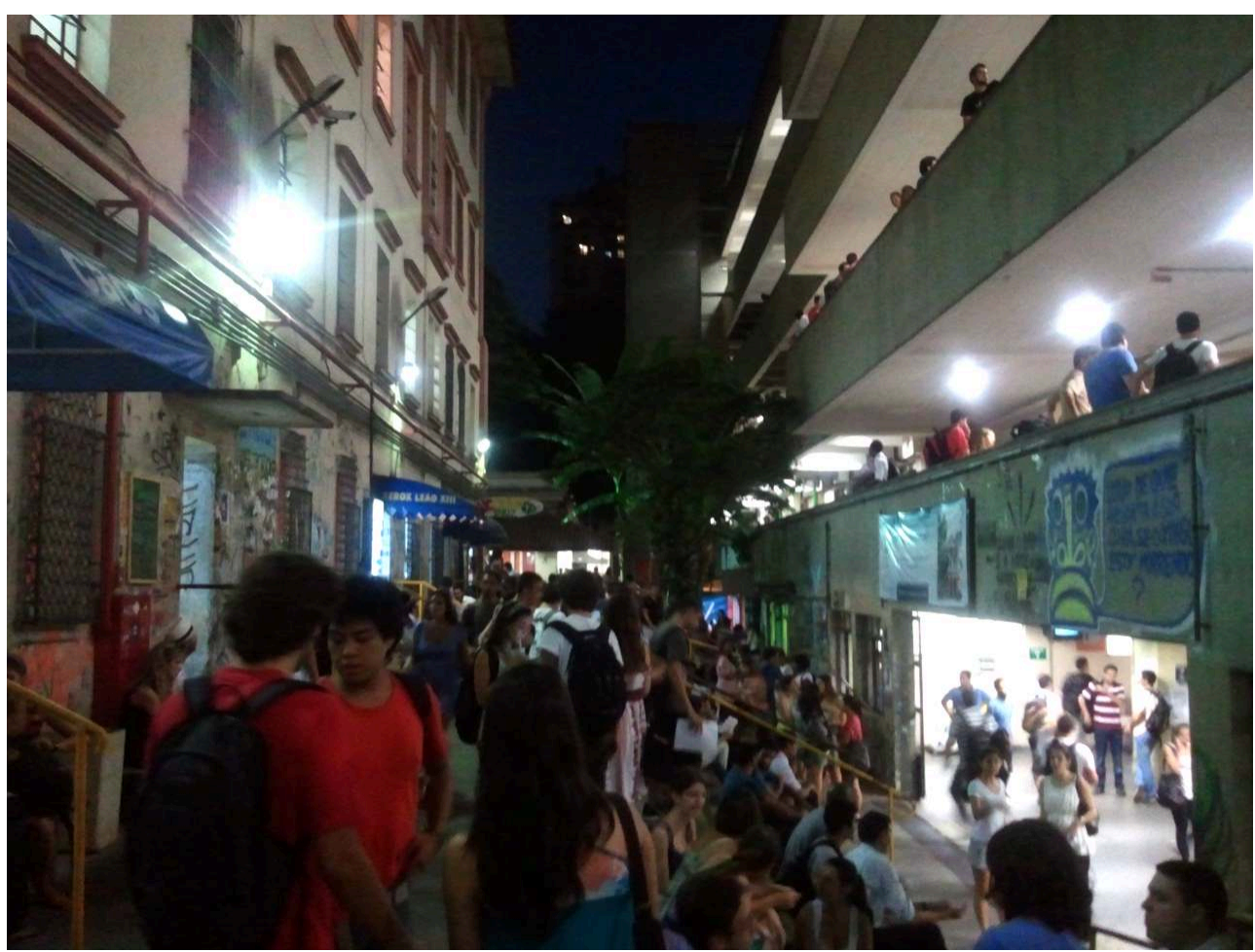

João Sette W. Ferreira

Le troisième type d'université, très récent, est le fruit des politiques néolibérales des années 1990, et notamment des politiques de privatisations du gouvernement Cardoso: ce sont les universités privées et payantes, véritables «boites commerciales ", qui aujourd'hui offrent d'un coté un plus grand accès à l'université, mais d'un autre coté sont d'une qualité scientifique des plus douteuses.

Il y a là une inversion assez perverse, qui reflète la logique de concentration des richesses qui marque la société brésilienne. L'école publique gratuite, de très mauvais niveau et délaissée depuis toujours, fréquentée par les plus pauvres, ne donne pas un niveau suffisant pour accéder aux prestigieuses universités publiques - et gratuites comme l'USP ou les universités fédérales. Ses élèves proviennent, plutôt, de l'élite dorée, qui a pu entrer dans les écoles privées (une école privée de haut niveau à São Paulo - du primaire au lycée - coûte environ 600 euros/mois par élève), et réussir les concours d'entrée à l'université publique. Jusque récemment, les plus démunis $n$ 'avaient accès à aucune université. Depuis l'autorisation de création d'un nombre élevé d'universités privées payantes, ils ont désormais accès à cette modalité, payante, mais de très mauvais niveau. On pourrait dire que l'enseignement supérieur au Brésil est malheureusement divisé en deux niveaux, un "vrai», celui des universités publiques et d'une bonne partie des communautaires (payantes et assez chères), et un "sous-niveau ", celui des universités payantes privées plus récentes, qui donnent un diplôme moins réputé et donc moins efficace pour l'entrée dans le marché de travail. Il y a évidemment beaucoup d'universités privées, et il ne faut pas généraliser, certaines réussissant à atteindre un niveau de meilleure qualité.

L'accès à ces universités payantes n'est pas généralisé aux plus pauvres, (le prix est situé entre 200 et 600 euros/mois, selon les universités et le public recherché), et vise 
surtout la nouvelle classe moyenne qui augmente avec la croissance économique du pays. Depuis le gouvernement Lula, un programme de bourses assez important, le Pro-Uni, a commencé à faire face à ce problème.

En France, la création des campus universitaires en périphérie depuis les années soixante a tantôt permis une réelle articulation au tissus urbain local tantôt provoqué la fermeture sur soi de l'espace universitaire. Quelle est l'expérience brésilienne?

João Sette Whiteker Ferreira : Les campi de ces universités - qui se distribuent dans tout le pays - sont en général situés dans la ville, et à cause du manque de possibilités foncières, occupent de grands terrains mais pas des campus gigantesques. Par contre, elles construisent sur ces terrains des immeubles assez imposants, recevant des milliers d'élèves par jour. Étant dépendantes de la publicité et de leur image, leurs immeubles sont souvent assez "criants", hauts en couleurs et très imposants. Les plus grands groupes (UNIP, Uninove) font des opérations immobilières importantes, et souvent achètent maison par maison des quadrilatères entiers, pour agrandir leurs propriétés foncières.

\section{Campus Uninove, Sao Paolo}

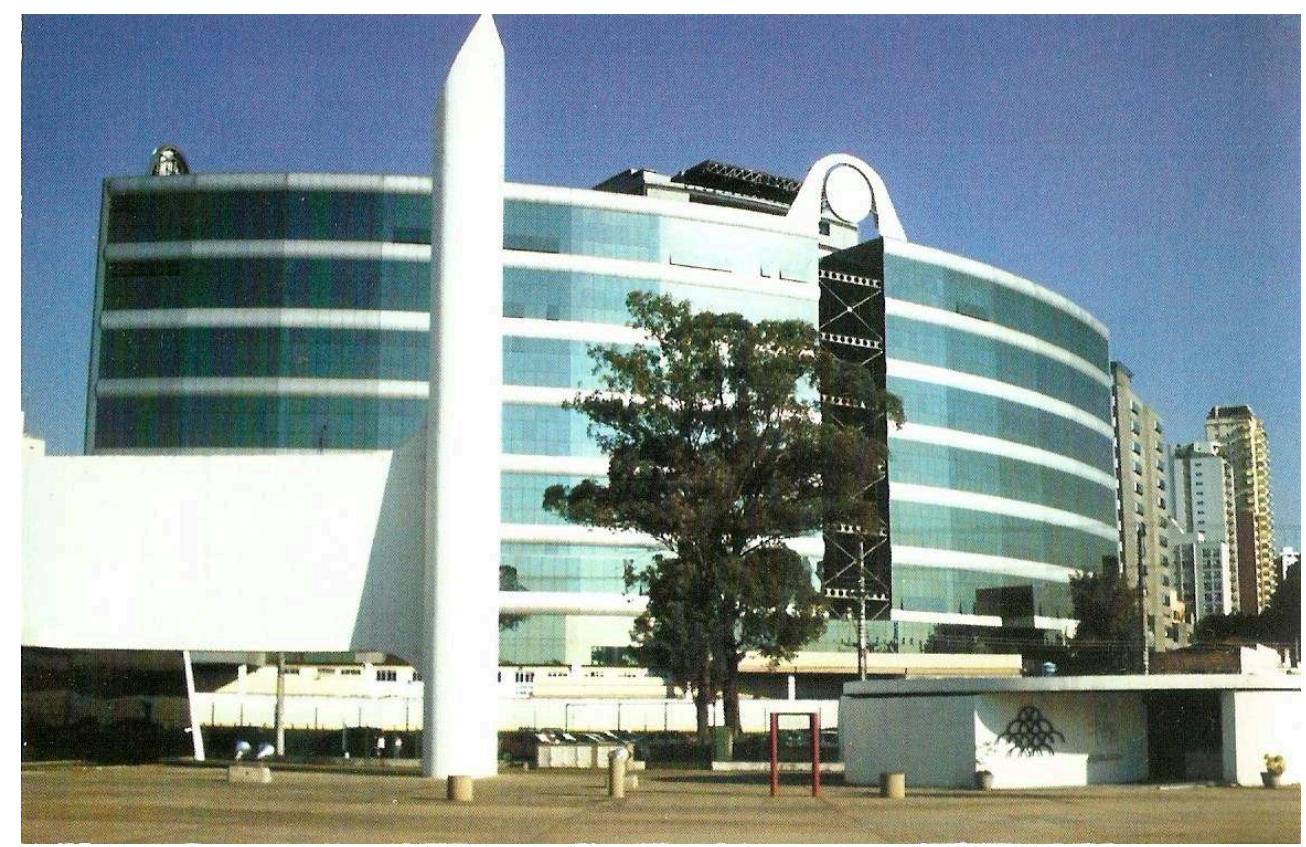

Felipe Mostarda - Wikimedia Commons/GNU Free Documentation Licence

Les grands campi des universités publiques, comme celui de l'université de São Paulo, ont souvent été créés durant la dictature (1964), ou avant même. L'USP a été créée en 1934, suite à la révolution paulista de 1932 et au désir de l'état de construire une position de leadership politique dans le futur. Elle regroupait ainsi administrativement les facultés plus anciennes, qui en général se maintinrent (jusqu 'à aujourd'hui) dans leurs immeubles originaux, éparpillés dans le centre-ville. Un projet de campus a été proposé dès cette époque, et un vaste terrain a été exproprié á cette fin, mais il a mis plusieurs décennies à se mettre en place. En fait, comme dans le cas de la majorité des campi des universités fédérales, c'est sous le régime militaire que ces aires furent réellement aménagées. 
Alors que ces espaces demanderaient des projets urbains assez sophistiqués pour créer, malgré l'ampleur de l'espace, des trajets urbains et des liaisons entre les unités à l'échelle des piétons, c'est exactement le contraire qui fut mis en pratique. En fait, le souci des militaires était justement d'éviter les possibles agglomérations $d$ 'étudiants. Les immeubles de ces campi sont donc généralement vastes et peu denses, avec des immeubles bien séparés les uns des autres, souvent de plusieurs centaines de mètres, sans aucune intégration. L'élite brésilienne, qui fréquentait et fréquente toujours ces universités, ayant des voitures, les liaisons entre facultés sont faites en automobile. Il y a des transports en commun internes assez précaires, très peu fréquents, et les autobus publics y entrent mais avec quelques lignes seulement. Il n'y a pas de commerce, sauf quelques petits établissements de restauration dans les facultés. Des immeubles de logements universitaires existent à l'USP, mais ne sont pas la règle dans les autres campi publics. De toute façon, ils sont peu nombreux, car les étudiants des universités publiques en général ont de quoi se loger. Récemment, des « places bancaires » ont été crées, sous la pression des banques, avec des agences bancaires. Ce sont des campi très beaux, très verts, mais aussi très déserts et peu urbains. Souvent, des équipements sportifs sont disponibles, comme le très beau centre sportif de l'USP, mais aussi isolés dans l'aire de la « ville universitaire ».

\section{Campus USP, Sao Paolo}

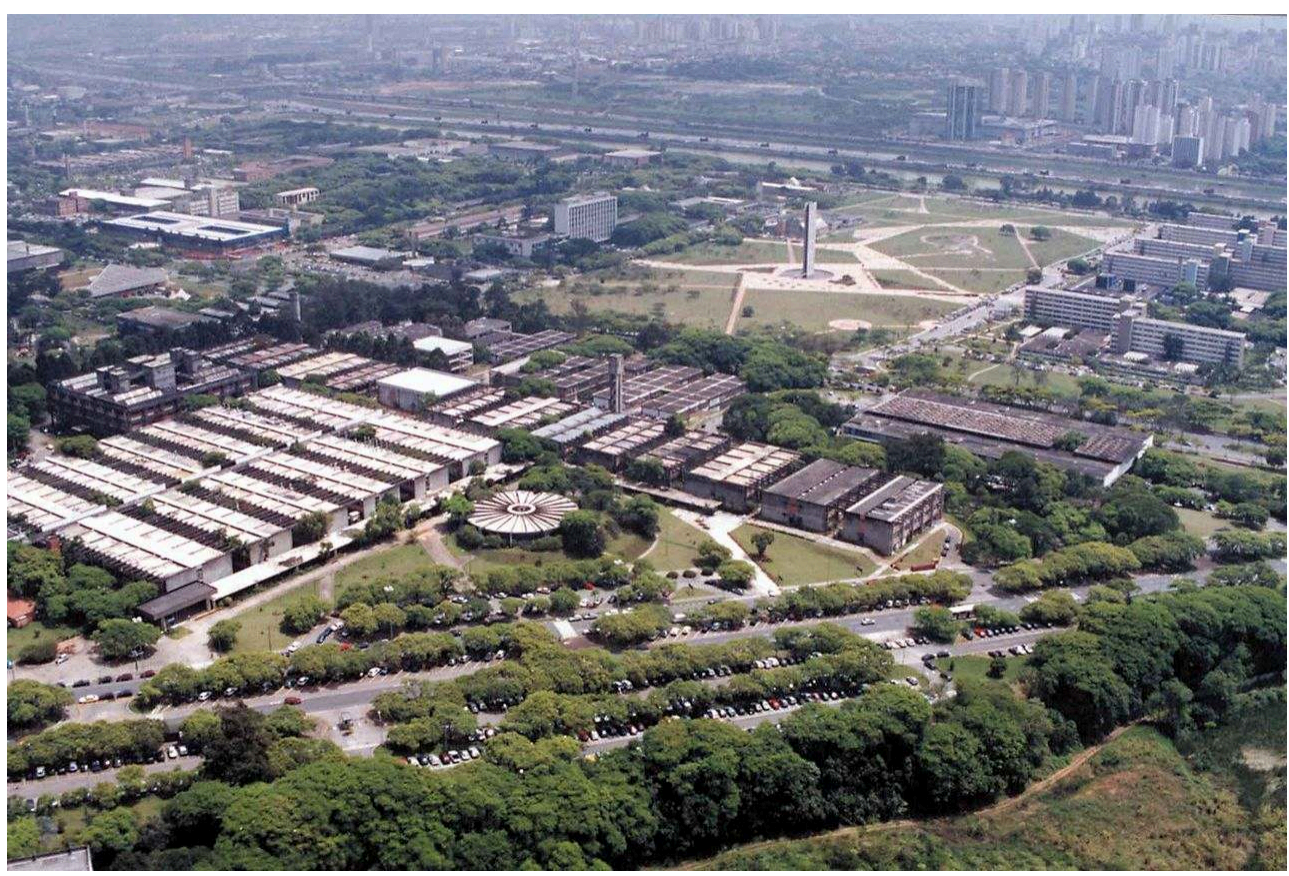

Hector Carvalho - Creative Commons

Durant le régime militaire, l'USP, ainsi que les autres campi d'universités fédérales à été assez souvent envahi par la police, créant souvent de violentes confrontations. De là est née, avec la démocratisation et l'autonomie universitaire, la défense des forces publiques d'entrer dans ces campi. Normalement, leur sécurité doit être assurée par des gardiens de l'université. Les immenses zones vides sont devenues des espaces propices à l'insécurité, même si leur entrée est contrôlée. Souvent encerclées par des favelas, les campi sont des lieux de tensions inhérentes. C'est l'une des raisons par lesquelles les gouvernements justifient l'entrée de la police. Mais le mouvement 
étudiant plus politisé, les accuse d'en profiter pour augmenter le contrôle social et réprimer les manifestations. Le campus de l'USP vit en ce moment une forte crise dans ce sens : en octobre, des policiers en civil ont arrêté dans le campus trois élèves qui fumaient de la marijuana, provocant une vive réaction et l'occupation du rectorat, en protestation contre la présence de la police. Le gouvernement a fait sortir les élèves avec une opération militaire disproportionnée, et les étudiants ont, en réponse, décrété une grève générale. Si les étudiants contestent la présence de la police militaire dans le campus (un héritage de la dictature contraire à l'autonomie universitaire), une majorité d'étudiants moins politisés, de classe moyenne et allant au campus en voiture, se déclarent très favorables à la présence de la police. Ce scénario a lieu dans un campus d'université d'état parce que c'est le gouvernement de São Paulo qui actuellement prend ce type de position au sujet du contrôle des campi, alors que le gouvernement fédéral, de profil bien plus progressiste, est dans une phase de grand respect envers l'autonomie des vastes campi des fédérales. Mais tout pourrait s'inverser, au gré des couleurs politiques des différents gouvernements dans les états fédéraux. En fait, c'est une discussion sans fin, qui reflète le caractère inapproprié de ces vastes campi isolés dans les villes très urbanisées du Brésil. Une vraie dynamique de commerce, de services, de rythme de vie et de flux urbains, à eux seuls, pourraient apporter la sécurité demandée.

Ces universités reflètent souvent tout l'élitisme qui structure les logiques sociales brésiliennes. Il y a quelques années, une ligne de métro fut construite, passant dans la région du campus de l'USP. Une opportunité rêvée d'ouvrir cette aire à la ville, de la rendre accessible à tous, grâce à un transport public de masse encore si rare dans la ville. Cependant, les décisions de cabinet, aussi bien du côté de l'université que du côté du gouvernement et du métro, en ont voulu autrement. La logique élitiste, qui voit ces grandes surfaces publiques comme la propriété privée de quelques privilégiés, a fait en sorte de construire la station de métro à un kilomètre de l'entrée principale. Ceux qui doivent aller au campus en métro, les plus pauvres, doivent encore prendre un bus payant jusqu'au portail d'entrée, et là attendre, longtemps, le passage du bus circulaire intérieur, gratuit, de l'université. Ce genre de décision met à nu les logiques des politiques publiques au Brésil, et montrent que les tensions dans les vastes campi universitaires sont encore très loin d'être résolues.

\section{NOTES}

1. En 1808 le Brésil est devenu le siège de l'empire portugais, lorsque le futur roi du Portugal, Jean VI s'y réfugie après l'invasion de son pays par les troupes de Napoléon $1^{\mathrm{er}}$.

2. Par exemple: l'université d'état de São Paulo occupe 100 hectares ou encore l'université fédérale de Brasilia occupe 180 hectares. 


\section{AUTEURS}

\section{JOÃO SETTE WHITAKER FERREIRA}

Architecte-urbaniste, économiste, détenteur d'une maitrise en en Science Politique, et PHD en Urbanisme. Professeur à la Faculté d'Architecture et Urbanisme de l'Université de São Paulo, Brésil, ou il est aussi Coordinateur du Laboratoire sur le Logement Social et les établissements humains (LabHab-FAUUSP). Professeur également à l'Université Mackenzie, à São Paulo, Brésil. Auteur de livres, articles et ouvrages sur la question urbaine et du logement au Brésil, il est aussi consultant dans le même domaine.

\section{CAPUCINE BOIDIN}

Maître de conférences en anthropologie à l'Université Sorbonne Nouvelle Paris 3, IHEAL (Institut des Hautes Etudes d'Amérique Latine). Chargée de cours de guarani à l'INALCO (Institut National des Langues et Civilisations Orientales), ses axes de recherche portent sur le métissage, le genre, les mémoires de guerre. Elle a publié en 2010 un numéro des Cahiers des Amériques latines sur le tournant décolonial.

http://www.ladocumentationfrancaise.fr/catalogue/3303332400621/index.shtml ; http:// www.iheal.univ-paris3.fr/spip.php?article1041 